\section{Using genetic signatures to better classify spinal neurons}

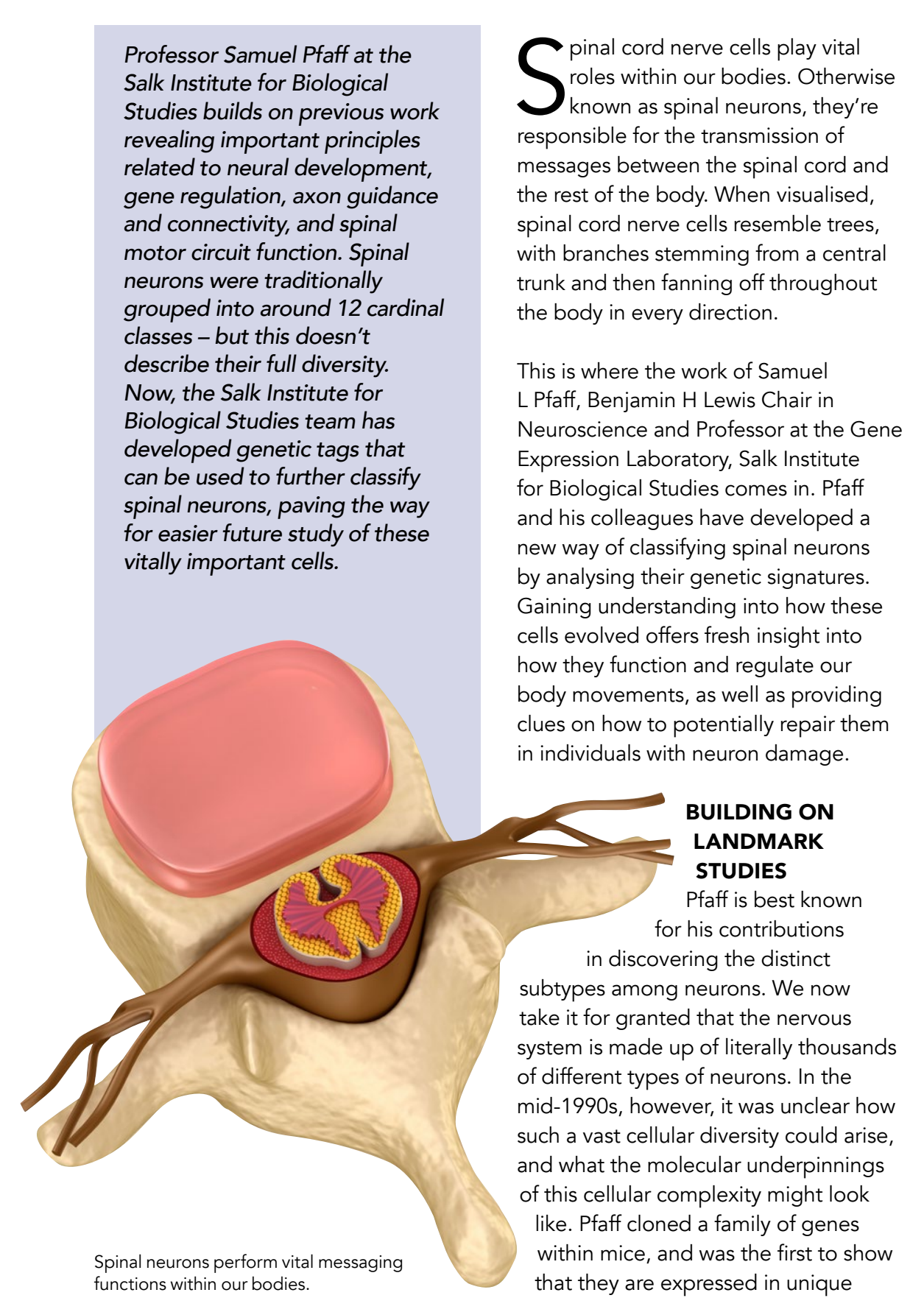

combinatorial arrays. These early landmark studies helped to define the anterent subtypes of motor neurons

His most recent work, recently published in the prestigious journal Science, expands on neuron classification even further.

WHY ARE SPINAL NEURONS SO IMPORTANT? Spinal neurons perform a vital messaging function within our bodies. When they're not functioning properly, there's no way for our brains to tell our muscles how and when to move. Put simply, if the messenger in the midale is out of action, communication becomes impossibl.

The spinal cord is an integral part of the body's central nervous system, and Something as simple as picking up glass, or typing on a keyboard, relies on huge amounts of sensory and motor information being conveyed to and from the brain via spinal neurons.

People with damaged spinal neurons can become paralysed. Their brains function normally but they are unable to make their bodies move in the way they want to, or even move at all. There are also a group of debilitating illnesses, known as neurodegenerative diseases, where the health and function of these cells are gradually worn down over time. Any research that can help people hrough accidents through accidents or who experience

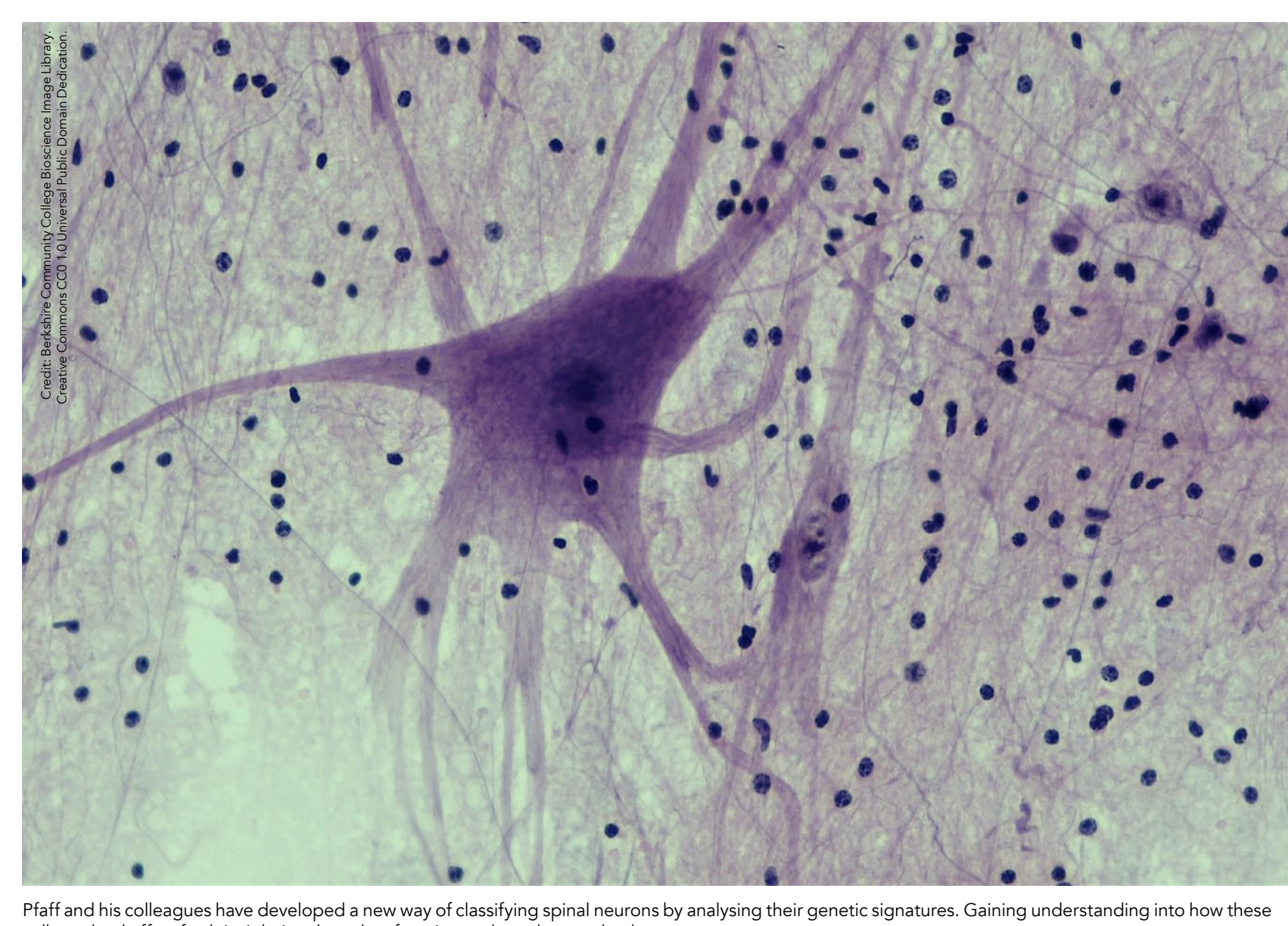

Pfaff and his colleagues have developed a new way of classifying spinal neurons by analysing
cells evolved offers fresh insight into how they function and regulate our body movements.

valuable, and of extreme importance to the medical community.

\section{HOW ARE SPINAL}

NEURONS CLASSIFIED? For some time, scientists have sorted spinal n'. This into so-called 'cardina classes'. This classification system is mainly based on where in the appear However there is significant diversity among the nurons within these cardinal classes.

It's an important goal to classify spinal neurons further than this, as it could help with understanding how the spinal cord neurons control movements and what goes wrong in neurodegenerative diseases or spinal cord injuries. Cardina classes are useful, but ultimately

incomplete when it com
spinal neuron diversity.

Previous attempts to diversify these cells further have been challenging, until Pfaf and his colleagues harnessed the power of single-cell genetic analysis to show that spinal gunetic andysis to show than previousty

\section{NEW DISCOVERIES}

The key to being able to further classify spinal neurons was gaining a better history. In a recent study, Pfaff and his colleagues discovered distinct genetic signatures that can be used to classify cells beyond the cardinal classes.

Using single-cell RNA sequencing technologies, almost 7,000 different

A study like this provides the first molecular handles for scientists to go in and study the function of spinal cord neurons more precisely than ever before. spinal neurons from mice were analysed. The aim was to see the differences in which genes were being activated, then use this data to try and group the neuron into closely related clusters. Essentially, it was similar to building an evolution ree of closely related organisms.

The researchers hoped to discover this evolutionary history by looking for cons different neural subtypes.

\section{CHARACTERISING SPINAL} NEURAL CIRCUITS Pfaff's lab has also made additional significant strides in our understanding of motor neuron gene regulation and axon guidance. His lab has conducted a series of studies that unravelled the spatial and temporal genetic-control mechanisms that establish motor neuron connectivity. These studies have helped the scientific community to understand how a relatively small number of signalling proteins contribute to the enormous wiring complexity of the 
Most recently his group has uncovered small RNA (microRNA) that is selectively microRNA is critical for maintaining neuromuscular synapses (messeges) and the survival of these neurons, and once levels drop below a critical threshold motor degeneration occurs. These new findings might help to understand why motor neuron degeneration oc amyotrophic lateral sclerosis (ALS).

\section{NEW GENETIC SIGNATURES} By identifying genetic relationships shared across neuronal populations in the spinal cord, the researchers uncovered an orderly series of neuronal systems. Firstly, the neuron components comprising sensoy having profoundly differed as having profoundly different genetic relay information about our limbs back to the spinal cord and prompt us to pull away reflexively when we touch painful stimuli - for example, when your hand automatically flinches away from touching a hot frying pan Motor circuitry enables us to do things like swing our arms while running to help maintain balance. Despite the heterogeneity of the neurons comprising sensory and motor circuits, the researchers identified simple gen patterns that distinguish these two

The more surprising division came when the researchers further analysed the split into two distinct a new genetic marker. When the tean used a biological dye to visualise cells belonging to each group in the spina cord, it became clear that the markers differentiated neurons based on whether they had long-range or short-range connections in the body.

Further experiments then revealed that the genetic patterns specific to long-range and short-range properties were common across all the cardinal classes tested. Notably, this study could open up exciting possibilities for the future treatment of spinal cord injuries. Pfaff explains. A study like this provides to go in and suand to for scientists cord in and study the fuscion or spinat

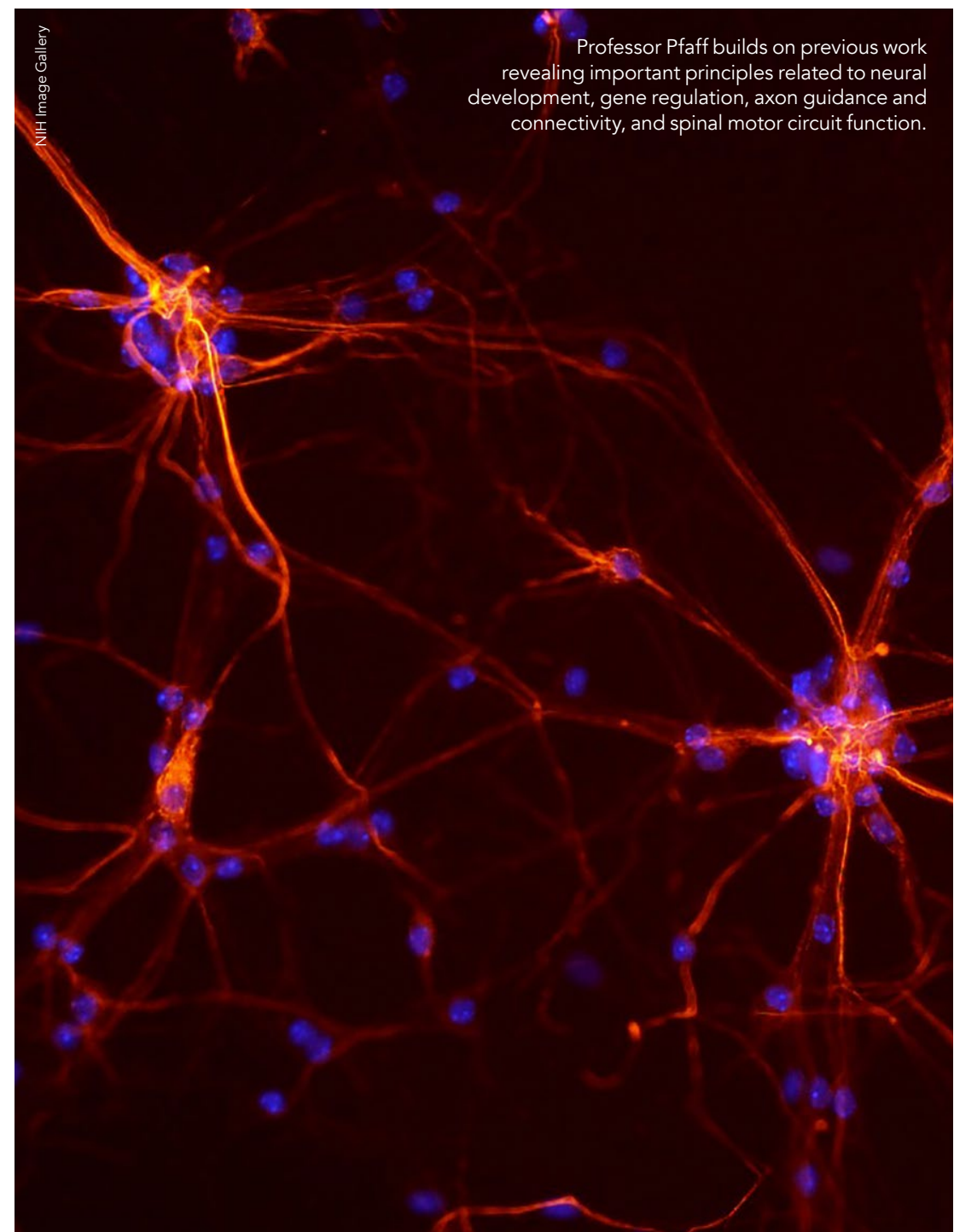

Thanks to the new findings, just two genetic tags are now needed to flag very specific populations of neurons.

way than they ever have before. This While the study was carried out in mice, also has implicating cord injuries.'

FUTURE OUTLOOK In the past, its researchers to use genetic tags to narrow down the one particular neuro type they wished to study, as using many markers is technically challenging. Thanks to these new findings, just two genetic tags are now needed to flag very specific populations of neurons Firstly, the previously known tag for cardinal class and secondly, the newly discover gentic tag for longrange the researchers have reason to predict that the same genetic patterns would with spinal cords. This could help when researchers are studying which groups of neurons are affected by spinal cord injury or neurodegenerative disease in humans.

It could also one day help to pave the way for research into how to regrow those specific cells in affected chasiduals. Having an easier way to classify and research neurons could lead medical field.

\section{Behind the Research} Professor Samuel L Pfaff

E: pfaff@salk.edu T: +18584534100 ×2018 (office) W: www.salk.edu/scientist/samuel-pfaff

\section{Research Objectives}

Professor Samuel Pfaff's lab used a novel bioinformatic patroch

\section{Detail}

\section{Address}

The Biological Studies

Gene Expression Laboratory

La Jolla CA 92109 USA

Dr Samuel L Pfaff is the Benjamin $\mathrm{H}$ Lewis Chair in Neuroscience and Professor in the Gene Expression Laboratory at the Salk Institute for Biological Studies. He aldo holds appointments with the Biology, Bioengineering, and Neuroscience programmes at the University of California, San Diego. He received his BA in biology in Carleton College (Northfield MN) and his lhD Berkeley. He completed postdoctoral training at Vanderb University with William Taylor on gene regulation followed by Columbia University with Thomas Jessell on neural development. He is the recipient of the Javits Neuroscience Anestigar Award, McKnightSchor Awed, PEW Schold

Funding

his research was supported by the Benjamin $\mathrm{H}$ Lewis chair Jience, Sol Goldman Charitable Trust, Christopher Dana Reeve Fandion, and National Institutes of

Health (1 U19 NS112959-01).

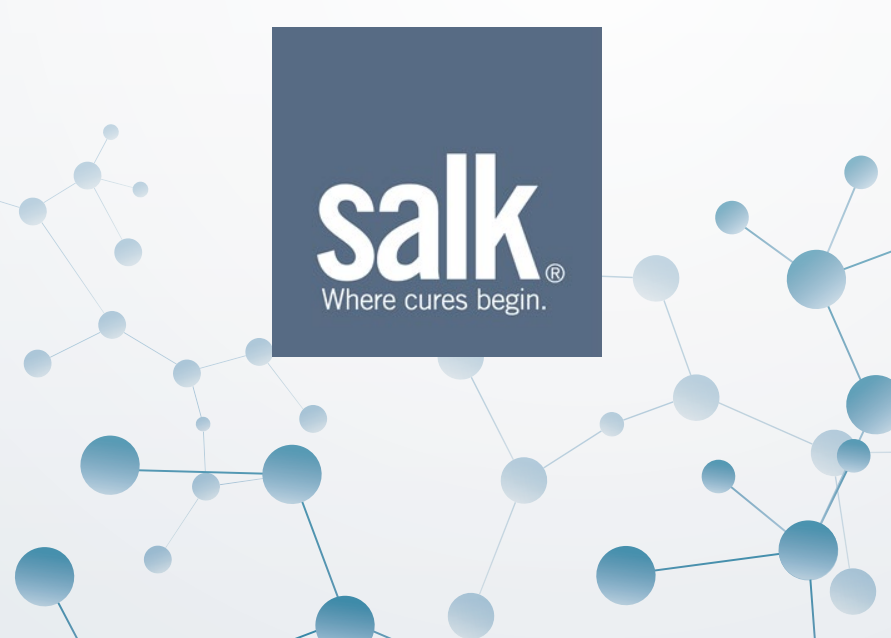

\section{References}

Osseward, P, (2021) Conserved genetic signatures parcellate cardinal spinal neuron classes into local and projection subsets. Science. 372, 6540: 385-393. doi.10.1126/science.abe0690

Amin, ND, Senturk, A, Costaguta, G, Driscoli, S, O'Leary, B, Bonanomi, D, Pfaff, SL, (2021) A hidden threshold in motor neuron gene networks for suw Aval revealed by modulation of neuron.2021.07.028

\section{Personal Response}

What do you believe will be the most interesting practical application of this research in the future? II With a relatively simple toolset of molecular markers that can be used in pairwise combinations, researchers can nownal neurons. A major research question that rens of to understand how spinal circuits integrate volitional and subconscious sensory commands and compute precise limb movements that involve many highly coordinated muscle groups. Scientists are now in a position to selectively study the function of very discrete neuron populations to understand how complex motor circuits function. The principles that underlie spinal motor circuit computatio will help to better understand mechanistically how behaviour is controlled.
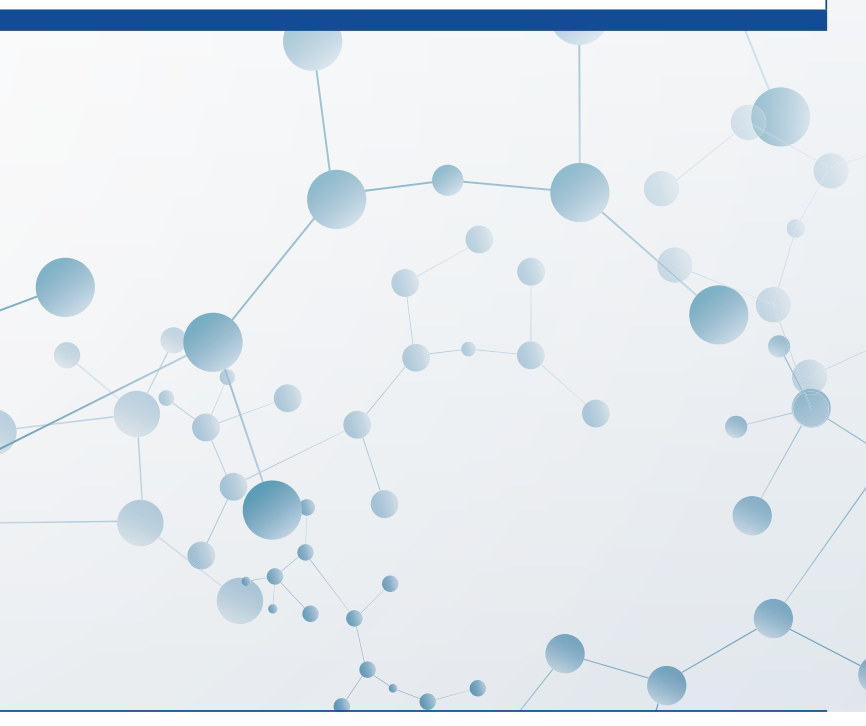